\title{
Extração de compostos fenólicos do noni (Morinda citrifolia Linn) empregando energia ultrassônica
}

Extraction of noni phenolic compounds (Morinda citrifolia Linn) using ultrasonic energy

\author{
I. A. S. I. Lima ${ }^{1}$; I. A. Ribeiro후 T. S. Neris ${ }^{1} ;$ S. S. e Silva ${ }^{2} ;$ R. A. Loss ${ }^{1}$; S. F. \\ Guedes $^{1}$ \\ ${ }^{1}$ Faculdade de Arquitetura e Engenharias, Universidade do Estado de Mato Grosso, CEP:78390-000,Barra do Bugres- \\ MT, Brasil \\ ${ }^{2}$ Faculdade de Ciências Exatas e Tecnológicas, Universidade do Estado de Mato Grosso, CEP:78390-000, Barra do \\ Bugres-MT, Brasil
}

*sumariasousa@gamail.com

(Recebido em 18 de janeiro de 2018; aceito em 19 de abril de 2018)

\begin{abstract}
O noni (Morinda citrifolia Linn) é uma fruta conhecida pelo seu alto valor nutracêutico, em especial pelo seu potencial antioxidante, que sofre influência de acordo com a forma de consumo, in natura ou processado. Diante do exposto, o presente trabalho teve como objetivo quantificar o teor de compostos fenólicos totais presentes na biomassa desidratada do noni. Para o preparo da biomassa, os frutos sem sementes foram higienizados e triturados. Posteriormente, as amostras foram desidratadas a $60{ }^{\circ} \mathrm{C} \mathrm{em}$ estufas com circulação de ar forçada. As amostras foram submetidas a extração com etanol, incubadas ou não em banho ultrassônico, conforme condições do planejamento fatorial completo $2^{2}$, cujas variáveis foram o tempo e o pH. Ao final da extração, o solvente foi evaporado, o extrato diluído em $5 \mathrm{~mL}$ de água destilada e submetido a análise dos compostos fenólicos totais. Nas extrações incubadas com o banho ultrassônico observou-se que este contribuiu para o aumento da concentração de compostos fenólicos totais extraídos em comparação ao método de maceração. Além disso, a extração assistida por energia ultrassônica reduziu o tempo de extração de 240 para 15 minutos. Desta forma, o ultrassom pode ser considerado uma ferramenta útil para aumentar a transferência de massa no processo de extração de compostos fenólicos da biomassa desidratada do noni.
\end{abstract}

Palavras-chave: Morinda citrifolia Linn, atividade antioxidante, etanol

Noni (Morinda citrifolia Linn) is a fruit known for its high nutraceutical value, especially for its antioxidant potential, which is influenced according to the form of consumption, in natura or processed. In view of the above, the present work had as objective to quantify the content of total phenolic compounds present in the noni dehydrated biomass. To prepare the biomass, the seedless fruits were sanitized and ground. Subsequently, the samples were dehydrated at $60{ }^{\circ} \mathrm{C}$ in greenhouses with forced air circulation. The samples were extracted with ethanol, incubated or not in an ultrasonic bath, according to the conditions of the complete factorial design 22, whose variables were time and $\mathrm{pH}$. At the end of the extraction, the solvent was evaporated, the extract diluted in $5 \mathrm{~mL}$ of distilled water and subjected to analysis of the total phenolic compounds. In the extractions incubated with the ultrasonic bath it was observed that this contributed to the increase in the concentration of total phenolic compounds extracted in comparison to the maceration method. In addition, the extraction assisted by ultrasonic energy reduced the extraction time from 240 to 15 minutes. In this way, ultrasound can be considered a useful tool to increase mass transfer in the process of extracting phenolic compounds from dehydrated noni biomass.

Keywords: Morinda citrifolia Linn, antioxidant activity, ethanol

\section{INTRODUÇÃO}

A maior variedade de espécies de plantas no mundo localiza-se em países em desenvolvimento. No Brasil se encontram cerca de $67 \%$ dessas espécies, dentre elas o noni (Morinda citrifolia Linn), originário da Polinésia e Caribe. O noni é comumente encontrado nos Estados de São Paulo, Acre, Ceará, Minas Gerais, São Paulo, Pará, Sergipe, entre outras regiões [1].

O noni é um fruto que apresenta uma elevada variedade de substâncias bioativas e que conferem a ele, propriedades anti-inflamatórias, antioxidantes, anti-helmínticas [2]. Além de 
estimular de maneira benéfica o sistema imunológico, favorecendo a produção de macrófagos e linfócitos, agindo nas defesas do organismo e auxiliando no combate as bactérias, vírus e células cancerígenas [3]. O elevado teor de compostos fenólicos em substâncias antioxidantes de origem natural promove o melhoramento da estabilidade, além da capacidade de proteção do organismo contra processos oxidativos, tornando-se uma alternativa economicamente viável devido a sua fácil acessibilidade e abundância [3,4].

Em estudos realizados com o fruto do noni foram encontrados aproximadamente 200 componentes fitoquímicos, em especial compostos fenólicos, alcalóides e ácidos orgânicos. Neste sentido, a extração e utilização destes compostos oferecem uma nova opção para agregar valor ao noni, uma vez que os extratos obtidos podem ser usados como fonte de ingrediente no ramo alimentício, farmacêutico e cosmético $[4,5,6]$.

Métodos clássicos de extração (extração por agitação em leito) e não clássicos (extração assistida por micro-ondas, acelerada com a utilização de solventes, extração assistida por energia ultrassônica ou extração com fluido supercrítico) estão sendo aplicadas na extração de compostos bioativos residuais de vegetais, sendo reconhecido que os métodos não clássicos apresentam um menor tempo de extração, maior rendimento e extratos de melhor qualidade tecnológica [7, 8].

A técnica de extração assistida por ultrassom tem como principal característica as forças de cavitação, que envolvem a catástase de bolhas formados do meio aquoso durante o processo, gerando assim uma rápida compressão adiabática dos vapores e gases no interior das cavidades e bolhas, ou seja, é um processo que não permite a entrada nem saída da energia térmica gerada no sistema. E assim, tendo como consequências a produção de altas pressões e temperaturas, ocasionando em uma extração eficiente $[9,10,11]$.

Diante do exposto, o presente trabalho teve como objetivo avaliar o uso de energia ultrassônica no processo de extração de compostos fenólicos totais contidos na biomassa desidratada do noni.

\section{MATERIAL E MÉTODOS}

\section{Local da pesquisa}

O presente trabalho foi realizado junto ao Departamento de Engenharia de Alimentos da Universidade do Estado de Mato Grosso (UNEMAT), Campus de Barra do Bugres - MT. Este município encontra-se localizado na mesorregião sudoeste matogrossense, em uma região de transição de Biomas (Amazônia e Cerrado) onde o clima é bem característico, sendo classificado como tropical quente e sub-úmido, apresentando verões chuvosos (dezembro a março) e invernos secos com precipitação média anual em torno de $1.800 \mathrm{~mm}$ [12].

\section{Reagentes e padrões}

Os solventes orgânicos etanol, éter etílico e metanol foram obtidos da Synth Acessórios e Equipamentos para Laboratórios (Diadema, Brasil). O padrão de ácido gálico utilizado na elaboração das curvas de calibração e o carbonato de sódio usado na extração dos compostos fenólicos foram obtidos da Vetec Química Fina Ltda (Belo Horizonte, Brasil) e o Folin \& Ciocalteau da Sigma-Aldrich Corporation (St. Louis, U.S.A).

\section{Obtenção da amostra}

O noni foi coletado no estádio 3 de maturação na região Centro-oeste do Brasil no mês de novembro. Os frutos foram transportados até o Laboratório de Química, alocados em caixas de isopor e mantidos em temperatura ambiente. Para o preparo da amostra, os frutos foram limpos com detergente comercial em água corrente e imersos em solução de hipoclorito de sódio a 2,5\% (v/v) durante 20 minutos. A matéria-prima (polpa e casca, sem semente) foi triturada com auxílio de liquidificador, a fim de se obter uma mistura homogênea (biomassa). Posteriormente, a biomassa foi submetida a desidratação em estufa de circulação forçada Cienlab (CE-220/Brasil) a 
$60{ }^{\circ} \mathrm{C}$ por 12 horas. O produto desidratado foi triturado e armazenado em frascos de polietileno a $18^{\circ} \mathrm{C}$ até realização das análises.

\section{Extração de compostos fenólicos}

Para a extração de compostos fenólicos foram realizados ensaios pelo método convencional (maceração) e também pela aplicação de energia ultrassônica, empregando metanol como solvente. Para o método de maceração, a extração foi realizada conforme descrito por Mylonaki et al. (2008) [13], onde uma proporção de 1:4 de biomassa e solvente (m:v) foi submetida ao processo de extração, semiagitação ao abrigo da luz. Para a extração assistida por energia ultrassônica foi utilizado a proporção de 1:4 de amostra e solvente (m:v), porém, os ensaios foram incubados em banho ultrassônico (Quimis - Q335D, Brasil), com uma frequência de $40 \mathrm{kHz}$.

Para avaliar o efeito do $\mathrm{pH}$ e tempo de extração na obtenção de extratos com elevado teor de compostos fenólicos, foi utilizado um planejamento fatorial completo $2^{2}$, com três pontos centrais. $\mathrm{O}$ mesmo planejamento foi utilizado tanto para o processo de maceração quanto para o assistido por ultrassom, porém com redução de tempo de extração para este último processo (Tabela 1).

Tabela 1: Níveis e variáveis do planejamento fatorial completo $2^{2}$, usado na extração de compostos fenólicos do noni

\begin{tabular}{llll}
\hline \multirow{2}{*}{ Níveis } & $\mathbf{p H}$ & Maceração & Banho ultrassônico \\
\cline { 3 - 4 } & 3 & 2 & Tempo (horas) \\
\hline-1 & 5 & 4 & 15 \\
0 & 7 & 6 & 30 \\
1 & & & 45 \\
\hline
\end{tabular}

Após a extração, a solução foi filtrada, o solvente evaporado em banho termostatizado a $60{ }^{\circ} \mathrm{C}$ e o extrato diluído em $5 \mathrm{~mL}$ de água destilada. A solução foi mantida congelada a $-20{ }^{\circ} \mathrm{C}$ até as análises. O processo de extração foi avaliado em função do teor de compostos fenólicos totais, cujas análises foram realizadas em triplicata e o resultado expresso em média e desvio padrão.

\section{Determinação dos compostos fenólicos totais}

Os compostos fenólicos totais foram avaliados conforme metodologia descrita por Machado et al. (2013) [14]. Uma alíquota de $750 \mu \mathrm{L}$ do extrato foi transferida para um tubo com tampa, sendo adicionados $250 \mu \mathrm{L}$ do reagente Folin \& Ciocalteau (3:10 em meio aquoso). A solução foi mantida em repouso de 3 a 8 minutos, adicionados $500 \mu \mathrm{L}$ de carbonato de sódio a $24 \%(\mathrm{~m} / \mathrm{v})$ e deixados em repouso por duas horas, ao abrigo da luz. Na sequência, foi realizada a leitura da absorbância a $760 \mathrm{~nm}$ em um espectrofotômetro (Belphotonics - SF200DM, Itália). Para maior confiabilidade, um reagente branco foi conduzido nas mesmas condições.

Para a quantificação dos compostos fenólicos foi construída uma curva analítica contendo 1 a 12,5 $\mu \mathrm{g}$. $\mathrm{mL}^{-1}$ de ácido gálico e os resultados foram expressos em mg equivalente em ácido gálico $\left(\mathrm{GAE} . \mathrm{g}^{-1}\right)$ de amostra desidratada $\left(\mathrm{y}=0,058 \mathrm{x}+0,0344, \mathrm{R}^{2}=0,9983\right)$.

\section{Análise estatística}

Os resultados dos planejamentos experimentais foram analisados empregando o software Assistat 7.7 (Licença gratuita). Sendo que, para a comparação entre as médias dos diferentes métodos de extração foi empregado o teste de Tukey e para comparação das médias de todos os ensaios de um mesmo método foi usado o teste de Duncan. A influência das variáveis na extração dos compostos fenólicos totais do noni foi avaliada através da Análise de Variância (ANOVA) e t teste. 


\section{RESULTADOS E DISCUSSÃO}

\section{Extração de compostos fenólicos}

De acordo com Machado et al. (2013) [14], diversas variáveis influenciam diretamente na extração dos compostos fenólicos de frutas e hortaliças, como $\mathrm{pH}$ e tempo de contato, de forma que estes podem contribuir para o aumento e manutenção, ou para obtenção de baixas concentrações dos compostos bioativos. Dessa forma, os parâmetros avaliados neste trabalho já foram descritos na literatura e usados em ensaios para avaliar a eficiência de extração dos compostos fenólicos $[15,16,17]$

Os resultados da extração dos compostos fenólicos usando o método convencional (maceração) e a extração assistida por ultrassom, nas diferentes condições de pH e tempo estão apresentados na Tabela 2.

Tabela 2: Planejamento fatorial completo $2^{2}$, usado na extração de compostos fenólicos do noni. Os resultados foram expressos em mg equivalente em ácido gálico (GAE. $\left.g^{-1}\right)$ de amostra desidratada.

\begin{tabular}{cccccc} 
Ensaios & pH & \multirow{2}{*}{$\begin{array}{c}\text { Tempo } \\
\text { (horas) }\end{array}$} & $\begin{array}{c}\text { Tempo } \\
\text { (minutos) }\end{array}$ & Maceração & $\begin{array}{c}\text { manho } \\
\text { ultrassônico }\end{array}$ \\
\hline 1 & $-1(3)$ & $-1(2)$ & $-1(15)$ & $74,33 \pm 0,88^{\mathrm{aA}}$ & $167,93 \pm 4,22^{\mathrm{bB}}$ \\
2 & $1(7)$ & $-1(6)$ & $-1(45)$ & $73,72 \pm 4,21^{\mathrm{aA}}$ & $170,31 \pm 9,47^{\mathrm{bB}}$ \\
3 & $-1(3)$ & $1(6)$ & $1(15)$ & $69,75 \pm 1,91^{\mathrm{aA}}$ & $163,90 \pm 6,42^{\mathrm{bB}}$ \\
4 & $1(7)$ & $1(2)$ & $1(45)$ & $77,55 \pm 7,27^{\mathrm{aA}}$ & $165,17 \pm 0,95^{\mathrm{bB}}$ \\
5 & $0(5)$ & $0(4)$ & $0(30)$ & $85,50 \pm 2,57^{\mathrm{aA}}$ & $164,49 \pm 9,44^{\mathrm{bB}}$ \\
6 & $0(5)$ & $0(4)$ & $0(30)$ & $83,99 \pm 1,42^{\mathrm{aA}}$ & $166,13 \pm 3,97^{\mathrm{bB}}$ \\
7 & $0(5)$ & $0(4)$ & $0(30)$ & $77,64 \pm 2,18^{\mathrm{aA}}$ & $165,41 \pm 2,38^{\mathrm{bB}}$ \\
\hline
\end{tabular}

Letras minúsculas iguais, na mesma linha, as médias não diferem entre si pelo teste de Tukey ao nível de probabilidade de $5 \%(\mathrm{p}<0,05)$. Letras maiúsculas iguais na mesma coluna, as médias não diferem entre si pelo teste de Duncan ao nível de probabilidade de $5 \%(\mathrm{p}<0,05)$.

O banho ultrassônico contribuiu para o aumento da concentração de compostos fenólicos totais extraídos da biomassa de noni desidratada, uma vez que, independentemente das condições operacionais, a utilização dessa ferramenta, dobrou a concentração de compostos fenólicos em relação ao método de maceração (Tabela 2). Além disso, as quantidades de compostos fenólicos extraídos pelo método de maceração foram estatisticamente diferentes, pelo teste de Tukey, das quantidades obtidas pela extração assistida energia ultrassônica.

Na Tabela 2 também é possível observar que extração assistida por energia ultrassônica reduziu o tempo de extração. Pelo método de maceração, a maior concentração de fenólicos totais foi obtida nas condições operacionais utilizadas nos pontos centrais (ensaios 5, 6 e 7), cuja média foi de 82,38 $\pm 4,17 \mathrm{mgGAE} / 100 \mathrm{~g}$ e, onde o $\mathrm{pH}$ do solvente de extração foi ajustado para 5,0 e o tempo de maceração foi de $4 \mathrm{~h}$. Sousa-Sartori et al. (2013) [18] avaliaram a influência do pH na extração de compostos fenólicos totais de partes aéreas da cana-de-açúcar e também observaram que as maiores capacidades antioxidante foram alcançadas em ensaios realizados em $\mathrm{pH} 5,0$, sendo que o mesmo foi observado por Murakami (2010) [19] na extração de compostos fenólicos de folhas de erva mate.

Nos ensaios empregando banho ultrassônico, os ensaios 1 e 2 obtiveram a maior concentração de fenólicos (170,31 e 167,93 mgGAE/100g, respectivamente). Desta forma, é plausível considerar que o ultrassom reduziu o tempo de extração de 240 minutos (maceração) para 15 minutos (banho ultrassônico). A redução no tempo de extração é vantajosa no ponto de vista econômico, uma vez que reduz o gasto energético envolvido no processo. Além disso, longos períodos de extração aumentam possibilidade de oxidação dos fenólicos $[14,15]$. A redução do tempo de extração com o uso de ultrassom também foi observada por Silva et al. (2016) [20] na extração de compostos fenólico na lichia.

Pela Tabela 2 também é possível observar que, de acordo com o teste de Duncan, as concentrações de compostos fenólicos não diferem entre si nas diferentes condições 
experimentais usadas no planejamento fatorial, em ambas as extrações (maceração e extração com banho ultrassônico). Como os ensaios pelo método de maceração foram conduzidos para fins comparativos, os seus resultados não foram discutidos exaustivamente. Já os ensaios incubados em banho ultrassônico serão melhor explorados.

Apesar das diferentes condições empregadas nos sete ensaios não terem apresentado diferença estatística, observa-se que independente do $\mathrm{pH}$, quando o tempo de extração aumenta, o teor de compostos fenólicos reduziu, indicando que a variável tempo exerce uma influência maior na extração dos compostos fenólicos do noni. Essa influência pode ser observada no t test (Tabela 3 ), onde se observa que a média e a variável tempo foram estatisticamente significativas, com pvalor $\leq 0,05$, considerando o intervalo de $95 \%$ de confiança.

Tabela 3: Efeito da influência das variáveis (pH e tempo) na concentração de compostos fenólicos extraídos do noni

\begin{tabular}{|c|c|c|c|c|c|c|}
\hline Fatores & $\begin{array}{l}\text { Coeficiente } \\
\text { de } \\
\text { Regressão }\end{array}$ & $\begin{array}{l}\text { Erro } \\
\text { padrão }\end{array}$ & $\mathrm{t}(3)$ & $\mathrm{p}$-valor & $\begin{array}{c}\text { Estimativa } \\
\text { por } \\
\text { intervalo } \\
(95 \%) \\
\text { Limite } \\
\text { inferior } \\
\end{array}$ & $\begin{array}{c}\text { Estimativa } \\
\text { por } \\
\text { intervalo } \\
(95 \%) \\
\text { Limite } \\
\text { superior }\end{array}$ \\
\hline Média & 166,1900 & 0,494894 & 335,8092 & 0,000000 & 164,6150 & 167,7650 \\
\hline $\mathrm{pH}$ & 0,9125 & 0,654683 & 1,3938 & 0,257680 & $-1,1710$ & 2,9960 \\
\hline Tempo & $-2,2925$ & $\mathbf{0 , 6 5 4 6 8 3}$ & $-3,5017$ & $\mathbf{0 , 0 3 9 4 3 3}$ & $-4,3760$ & $-0,2090$ \\
\hline $\mathrm{pH} x$ tempo & $-0,2775$ & 0,654683 & $-0,4239$ & 0,700218 & $-2,3610$ & 1,8060 \\
\hline
\end{tabular}

A influência do tempo na extração de compostos fenólicos pode ser melhor visualizada no diagrama de Pareto (Figura 1), onde observa-se que apenas o tempo apresentou efeito estatisticamente significativo na extração de compostos fenólicos da biomassa de noni desidratada, sendo este efeito negativo, ou seja, menores tempos de extração conduziram a maiores concentrações de compostos fenólicos totais, o que corrobora com a discussão anterior dos resultados da Tabela 2 .

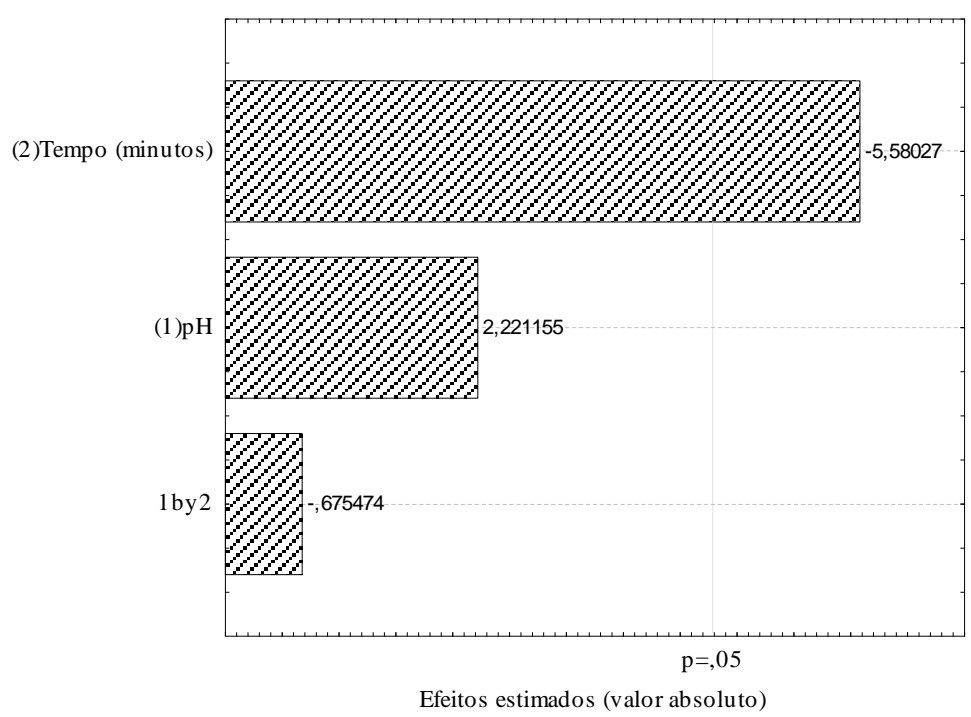

Figura 1: Diagrama de Pareto mostrando os efeitos das variáveis (pH e tempo) de extração na concentração de compostos fenólicos do noni empregando banho ultrassônico.

Como o pH não apresentou influência na extração dos compostos fenólicos e menores tempos conduziram a maiores concentrações de fenólicos, a condição escolhida como a mais favorável para a extração de compostos fenólicos do noni com ultrassom foi a condição do ensaio 
2 (pH 7,0 e 15 minutos). Com o objetivo de se observar melhor as condições experimentais que conduzem as maiores extrações de compostos fenólicos, foi gerado o gráfico superfície de resposta (Figura 2), onde pode-se visualizar uma tendência a maiores concentrações com a redução do tempo de contato e em pH menos ácido e alcalino (entre 5 e 7 ).

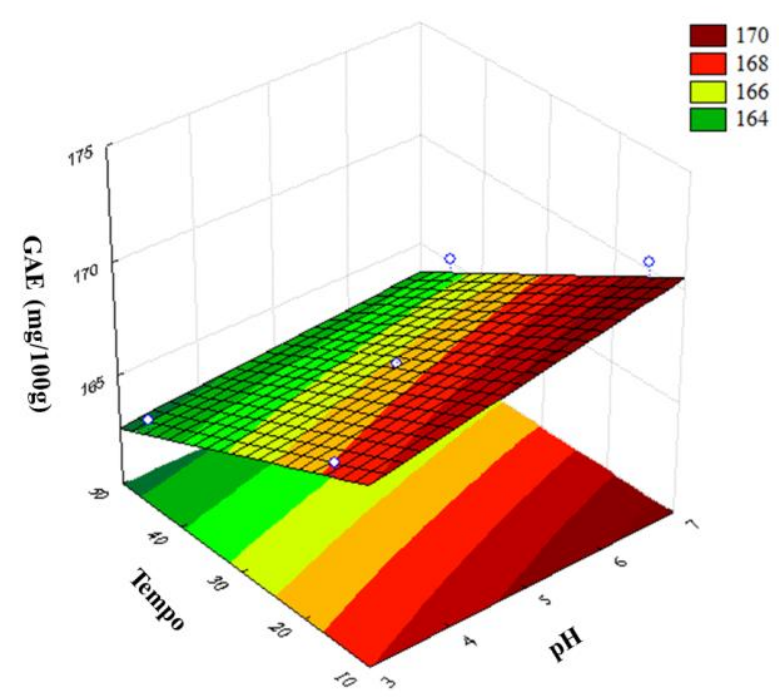

Figura 2: Superfície de resposta demostrando as melhores condições para a extração de compostos fenólicos totais do noni empregando banho ultrassônico.

\section{CONCLUSÃO}

A aplicação da energia ultrassônica apresentou diferença estatística quando comparado ao método de maceração. Das variáveis avaliadas na extração em banho ultrassônico, apenas o tempo influenciou na concentração dos compostos fenólicos totais do noni.

\section{AGRADECIMENTOS}

Os autores agradecem a Universidade do Estado de Mato Grosso (UNEMAT), pelo apoio estrutural e às agências de fomento à pesquisa pelo apoio financeiro, Fundação de Amparo à Pesquisa do Estado de Mato Grosso (FAPEMAT), por meio dos projetos de pesquisa $\mathrm{n}^{\circ}$ 210230/2015 e n⿳5十5980/2017 e Conselho Nacional de Desenvolvimento Científico e Tecnológico (CNPq), pela bolsa de Pesquisa DCR.

\section{REFERÊNCIAS BIBLIOGRÁFICAS}

1. Brasil. Ministério da Saúde. Secretaria de Atenção à Saúde. Departamento de Atenção Básica. Política nacional de práticas integrativas e complementares no SUS: atitude de ampliação de acesso / Ministério da Saúde. Secretaria de Atenção à Saúde. Departamento de Atenção Básica. - 2. ed. - Brasília: Ministério da Saúde; 2015.

2. Pimentel DD, Meira AMB, Araújo CRF, Peixoto MI. O uso de noni (Morinda citrofolia L.) por pacientes oncológicos: um estudo bibliográfico. Revista Saúde \& Ciência Online. 2016;5(1):37-44.

3. Mororó AVTP, Carvalho MJM, Araújo NTP, Cavalcante ALC, Catunda Júnior FEA, Bezerra SB, Cerqueira GS, Siqueira RMP. Morinda citrifolia (noni): uma revisão dos seus efeitos biológicos. Revista Intertox de Toxicologia, Risco Ambiental e Sociedade. 2017 Jun;10(2):46-61.

4. Costa AB, Oliveira AMC, Silva AMO, Filho-Mancini J, Lima A. Atividade antioxidante da polpa, casca e sementes do noni (Morinda citrifolia Linn). Revista Brasileira de Fruticultura. 2013 Jun;35(2):345-354. 
5. Sáyago-Ayerdi SG, Brenes A, Goñi I. Effect of grape antioxidant dietary fiber on the lipid oxidation of raw and cooked chicken hamburgers. Food Science Technology. 2009 Jun;42(5):971-976, doi:10.1016/j.lwt.2008.12.006

6. Almeida-Souza F, Taniwaki NN, Amaral AC, de Souza C da S, Calabrese K da S, Abreu-Silva AL. Ultrastructural Changes and Death of Leishmania infantum Promastigotes Induced by Morinda citrifolia Linn. Fruit (Noni) Juice Treatment. Evidence-Based Complementary and Alternative Medicine. 2016 Mai;2016:1- 9, doi:10.1155/2016/5063540

7. Palioto GF, Silva CFG, Mendes MP, Almeida VV, Rocha CLMSC, Tonin LTD. Composição centesimal, compostos bioativos e atividade antioxidante de frutos de Morinda citrifolia Linn (noni) cultivados no Paraná. Revista Brasileira de Plantas Medicinais. 2015 Jan-Mar;17(1):59-66, doi:10.1590/1983-084X/13_066

8. Tabaraki R, Rastgoo S. Comparison between conventional and ultrasound-assisted extractions of natural antioxidants from walnut green husk. Korean Journal of Chemical Engineering. 2014 Abr;31(4):676683, doi:10.1007/s11814-013-0279-1.

9. Ghitescu RE, Volf L, Carausu C, Bühlmann AM, Gilca IA, Popa VI. Optimization of ultrasoundassisted extraction of polyphenols from spruce wood bark. Ultrasonic Sonochemistry. 2015 Jan;22:535541, doi:10.1016/j.ultsonch.2014.07.013.

10. Ávila NS., Capote FP, Castro MDL. Ultrasound-assisted extraction and silylation prior to gaschromatography-mass spectrometry for the characterization of the triterpenic fraction in olives leaves. Journal of Chromatography A. 2007 Sep;1165(1-2):158-165, doi:10.1016/j.chroma.2007.07.039.

11. Fontana AR, Antoniolli A, Bottini R. Grape pomace as a sustainable source of bioactive compounds: extraction, characterization, and biotechnological applications of phenolics. Journal of Agricultural and Food Chemistry. 2013 Sep;61(38):8987-9003, doi:10.1021/jf402586f.

12. Instituto Brasileiro de Geografia e Estatística (IBGE). Censo 2010. Disponível em: <http://www.ibge.gov.br> Acesso em: 05 de jan. de 2018.

13. Mylonaki S, Kiassos E, Makris DP, Kefalas P. Optimisation of the extraction of olive (Olea europea) leaf phenolics using water/etanol-based solvente sstems and response surface methodology. Analytical and Bioanalytical Chemistry. 2008 Nov;392(5):977-985, doi:10.1007/s00216-008-2353-9.

14. Machado WM, Pereira AD, Marcon MV. Efeito do processamento e armazenamento em compostos fenólicos presentes em frutas e hortaliças. Publicatio UEPG: Ciências Exatas e da Terra, Agrárias e Engenharias. 2013 Jan-Jun;19(1):17-30, doi:10.5212/Publ.Exatas.v.19i1.0002.

15. Wang L, Weller CL. Recent advances in extraction of nutraceuticals from plants. Trends in Food Science \& Technology. 2006 Jun;17(6):300-312, doi:10.1016/j.tifs.2005.12.004.

16. González-Centeno MR, Knoerzer K, Sabarez H, Simal S, Rosselló C, Femenia A. Effect of acoustic frequency and power density on the aqueous ultrasonic-assisted extraction of grape pomace (Vitis vinifera L.) - A response surface approach. Ultrasonics Sonochemistry. 2014 Nov;21(6):2176-2184, doi:10.1016/j.ultsonch.2014.01.021.

17. Ricárdez OFM, Ruiz-Jiménez J, Lagunez-Rivera L, Castro MD. Fast ultrasound assisted extraction of polar (phenol) and nonpolar (lipids) fractions in Heteroth Inuloides Cass. Phytochemical Analysis. 2011 Nov-Dez;22(6):484-491, doi:10.1002/pca.1305.

18. Sousa-Sartori JA, Scalise C, Baptista AS, Lima RB, Aguiar CL. Parâmetros de influência na extração de compostos fenólicos de partes aéreas da cana-de-açúcar com atividade antioxidante total. Bioscience Journal. 2013 Mar-Abr;29(2):297-307.

19. Murakami ANN. Concentração de compostos fenólicos de extrato aquoso de folhas de erva-mate (Ilex paraguariensis A. St. Hil.) [dissertação]. Florianópolis: Universidade Federal de Santa Catarina- UFSC; 2010.

20. Silva C, Garcia VAS, Franciscato LMSS. Extração Assistida por Ultrassom de Compostos Bioativos das Cascas de Lichia (Litchi Chinensis Sonn.). Revista Ciências Exatas e Naturais. 2016 JanJun;18(1):81-96, doi:10.5935/RECEN.2016.01.07 